\title{
Rapid molecular TB diagnosis: evidence, policy making and global implementation of Xpert MTB/RIF
}

\author{
Karin Weyer ${ }^{1}$, Fuad Mirzayev ${ }^{1}$, Giovanni Battista Migliori², Wayne Van Gemert ${ }^{1}$, \\ Lia D’Ambrosio ${ }^{2}$, Matteo Zignol ${ }^{1}$, Katherine Floyd ${ }^{1}$, Rosella Centis², \\ Daniela M. Cirillo ${ }^{3}$, Enrico Tortoli ${ }^{3}$, Chris Gilpin', Jean de Dieu Iragena', \\ Dennis Falzon ${ }^{1}$ and Mario Raviglione ${ }^{1}$
}

\begin{abstract}
Affiliations:
'Stop TB Dept, World Health Organization, Geneva, Switzerland.

${ }^{2}$ WHO Collaborating Centre for TB and Lung Diseases, Fondazione S. Maugeri, Care and Research Institute, Tradate, and

${ }^{3}$ Emerging Pathogens Unit TB Supranational Reference Laboratory, San Raffaele Scientific Institute, Milan, Italy.
\end{abstract}

\section{Correspondence:}

G.B. Migliori, World Health Organization Collaborating Centre for TB and Lung Diseases, Fondazione S. Maugeri, Care and Research Institute, Via Roncaccio 16, 21049, Tradate, Italy.

E-mail: giovannibattista.miglioridfsm.it

ABSTRACT If tuberculosis (TB) is to be eliminated as a global health problem in the foreseeable future, improved detection of patients, earlier diagnosis and timely identification of rifampicin resistance will be critical. New diagnostics released in recent years have improved this perspective but they require investments in laboratory infrastructure, biosafety and staff specialisation beyond the means of many resource-constrained settings where most patients live. Xpert MTB/RIF, a new assay employing automated nucleic acid amplification to detect Mycobacterium tuberculosis, as well as mutations that confer rifampicin resistance, holds the promise to largely overcome these operational challenges. In this article we position Xpert MTB/RIF in today's TB diagnostic landscape and describe its additional potential as an adjunct to surveillance and surveys, taking into account considerations of pricing and ethics. In what could serve as a model for the future formulation of new policy on diagnostics, we trace the unique process by which the World Health Organization consulted international expertise and systematically assessed published evidence and freshly emerging experience from the field ahead of its endorsement of the Xpert MTB/RIF technology in 2010, summarise subsequent research findings and guidance on who to test and how, and provide perspectives on scaling up the new technology.

@ERSpublications

The most up-to-date evidence, policy-making and global implementation of the new diagnostic Xpert MTB/RIF http://ow.ly/ks9dN

This article has supplementary material available from www.erj.ersjournals.com

Received: Oct 042012 | Accepted after revision: Nov 082012 | First published online: Nov 222012

ERJ Open articles are open access and distributed under the terms of the Creative Commons Attribution NonCommercial Licence 3.0.

This article was modified in April 2016 to correct errors in the licence information. 


\section{Introduction}

With 8.7 million incident cases of tuberculosis (TB) and 1.4 million deaths estimated in 2011 [1], TB is a leading cause of morbidity and mortality worldwide. However, public health services globally reported only 5.8 million $(66 \%)$ of the estimated TB cases in 2011. Moreover, less than $5 \%$ of notified TB cases were tested for drug resistance [1], which is often diagnosed after prolonged diagnostic delays [2-4]. Of the 310000 notified new and re-retreatment cases with pulmonary TB estimated to have multidrug-resistant (MDR)-TB in 2011, just under 60000 (19\%) were reported to the World Health Organization (WHO) [1].

The main reasons for these gaps are inadequate diagnostic capacity and an over reliance on chest radiography and/or sputum smear microscopy as diagnostic tools. Patients with HIV-associated TB, those with sputum smear-negative and/or extrapulmonary disease, and drug-resistant TB patients are particularly affected by the failure of microscopy as a primary diagnostic tool. The "classical" diagnosis of HIVassociated and drug-resistant TB is complex, expensive, slow and technically demanding, relying on conventional culture and drug susceptibility testing (DST). The long delay (up to several weeks) required to obtain results has devastating consequences for patients who go undiagnosed (and therefore untreated or inappropriately treated), or are diagnosed too late [5].

Detecting more cases, detecting them early and rapidly identifying drug resistance are essential for improving individual patient health and avoiding transmission in the community. This requires universal access and early detection using contemporary tools and innovative strategies $[1,4,5]$.

The past decade has seen unprecedented growth in the TB diagnostic pipeline and accelerated efforts to establish the necessary laboratory infrastructure [5]. Nevertheless, although recommended by WHO, the latest generation liquid culture diagnostics and molecular line probe assays for rapid detection of MDR-TB have not yet solved the diagnostic dilemma in most resource-limited settings, largely due to the need for expensive laboratory infrastructure, extensive biosafety precautions and specialised staff [5]. A new rapid test that overcomes many of the current operational difficulties was recommended for use by WHO in December 2010: the Xpert MTB/RIF assay (Cepheid, Sunnyvale, CA, USA) is an automated, real-time nucleic acid amplification technology run on the multi-disease platform GeneXpert (Cepheid). The Xpert MTB/RIF assay represents a paradigm shift in the diagnosis of TB and drug-resistant TB by simultaneously detecting Mycobacterium tuberculosis and rifampicin resistance-conferring mutations in a closed system suitable for use outside conventional laboratory settings in less than $2 \mathrm{~h}$, directly from sputum samples $[6,7]$.

\section{Objectives}

This article has three primary objectives. The first is to describe the dynamic process followed by WHO in policy development for TB diagnostics, using the example of Xpert MTB/RIF assay as a pathfinder. The second is to summarise subsequent evidence on the use of Xpert MTB/RIF, clarify common misconceptions about the technology, and provide perspectives on the role of the assay in improved case detection and care delivery. The third is to summarise the relevance of the technology for TB prevalence surveys and drug resistance surveillance, its impact on case and treatment outcome definitions, and discuss issues around affordability, sustainability, ethics and research priorities.

\section{Methods}

Existing policy and guidance documents on Xpert MTB/RIF are summarised to illustrate the WHO policy formulation process for new TB diagnostics. Outcomes are presented from a Global Consultation organised by WHO immediately prior to endorsement of the assay. Experiences shared by early implementers of the assay during two subsequent WHO global meetings are also summarised.

For additional evidence on the Xpert MTB/RIF assay since WHO endorsement, active scanning of the emerging literature was performed. PubMed and EMBASE results were searched to find articles dealing with the Xpert MTB/RIF test. Combinations of the following search terms were used: "tuberculosis", "multidrug-resistant tuberculosis", "extensively drug-resistant tuberculosis", "Xpert MTB/RIF” and "rapid diagnosis". Although the search was not restricted to publications in English, articles not reporting an English summary were excluded.

Citations were independently screened by four investigators (K. Weyer, W. van Gemert, G.B. Migliori and R. Centis) by examining titles, abstracts and full articles to identify relevant studies, which are stored in the WHO database and regularly updated (last update September 21, 2012). Unpublished sources of data (multicentre laboratory validation and demonstration studies coordinated by FIND (Foundation for Innovative New Diagnostics; Geneva, Switzerland) and unpublished data from investigator-driven, singlecentre studies) shared with WHO at the time of policy development were also included. Although this perspective article includes all available evidence on Xpert MTB/RIF, the formal criteria for a systematic review were not followed. 


\section{Brief overview of the Xpert MTB/RIF technology}

The GeneXpert platform, launched in 2004, simplifies real-time PCR-based molecular testing by integrating and automating the key processes of sample preparation, amplification and detection. Core components of the system include the instrument, a personal computer, a barcode scanner and the software (fig. 1), together with disease-specific, single-use, disposable cartridges containing lyophilised reagents, buffers and washes. Target detection and characterisation is performed in real time using a six-colour laser detection device.

The Xpert MTB/RIF cartridge for the simultaneous detection of TB and rifampicin resistance was developed within 4 years, following a unique collaboration between academia and industry, brokered by FIND and financially supported by the US National Institutes of Health (Bethesda, MD, USA) and the Bill and Melinda Gates Foundation (Seattle, WA, USA) [8-13]. This collaboration serves as a blueprint for TB diagnostics development, consisting of clear end-user product specifications, adequate research funding, collaboration among academic partners on the core components of the technology, pooling of research resources, controlled clinical validation trials, large-scale field evaluations under well-designed operational research protocols, and a flexible response by industry engaging early on with FIND in negotiations on cost and preferential pricing.

The end-product was a fully automated, closed (and therefore safe) real-time PCR system, requiring basic not specialised laboratory infrastructure, operator skills or biosafety precautions [8-13]. The Xpert MTB/RIF assay employs five unique nucleic acid hybridisation probes (molecular beacons), each labelled with a coloured fluorophore responding to a specific target sequence within the rpoB gene of $M$. tuberculosis. More than $95 \%$ of mutations associated with rifampicin resistance occur in an 81-base pair core region of the rpoB (a bacterial RNA polymerase) gene and together these five molecular beacons encompass the entire core region. The generation of all five fluorescent colours during PCR amplification indicates the presence of rifampicinsusceptible M. tuberculosis, while any mutation in the core region prevents the binding of the respective molecular beacon, resulting in the absence of colour and indicating rifampicin resistance (fig. 2) [8-13].

\section{Evidence-based policy development}

In 2008, WHO adopted the international GRADE process (Grades of Recommendations Assessment, Development and Evaluation) for evidence synthesis and evaluation [14]. GRADE currently underpins all WHO recommendations and guidelines [15]. Recently refined for the evaluation of diagnostics [16], GRADE provides a systematic assessment of the quality of evidence underlying policy formulation, as well

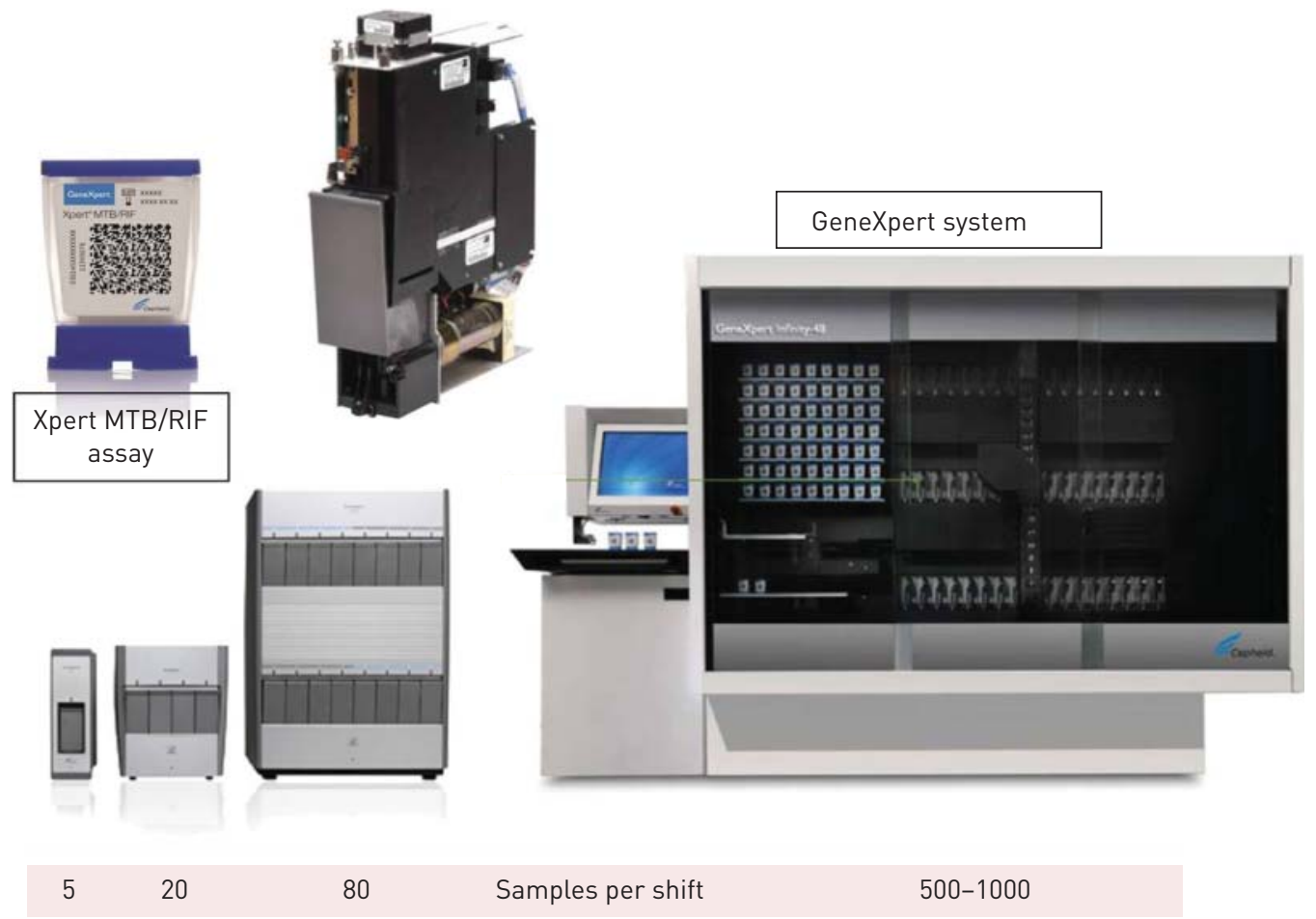

FIGURE 1 The GeneXpert system (personal communication; Foundation for Innovative New Diagnostics, Geneva, Switzerland). 


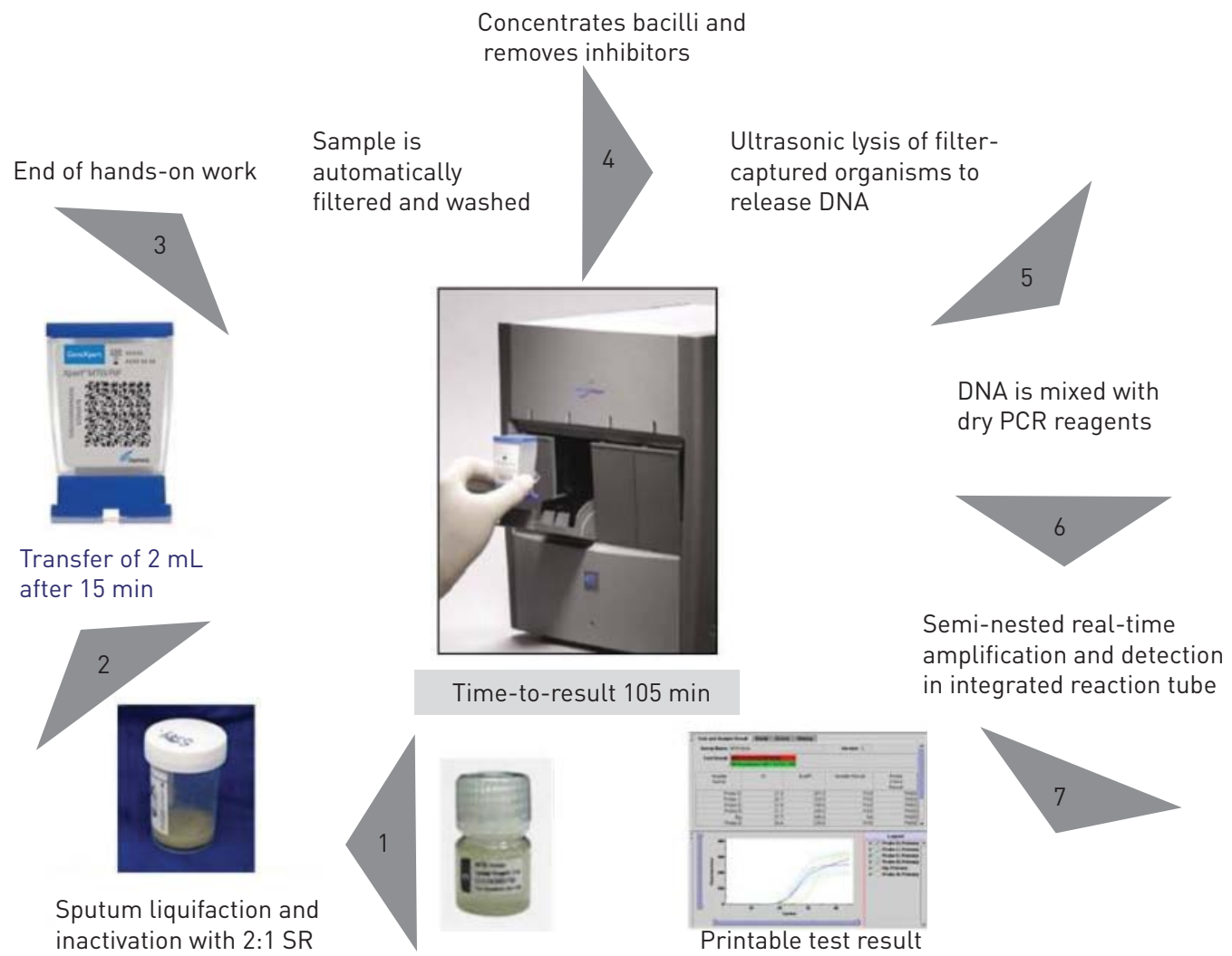

FIGURE 2 The step-by-step Xpert MTB/RIF assay process. SR: sterilising reagent. Reproduced from [6] with permission from the publisher.

as the strength of policy recommendations, aiming to achieve a balance between test performance, risks and benefits, and patient/public health impact $[14,16]$. The process is overseen by the WHO Guidelines Review Committee, which was specifically established for this purpose [15].

Figure 3 illustrates the structured approach to policy development on new TB diagnostics established in 2008 by the WHO Stop TB Department, while table 1 outlines the body of evidence required by WHO to proceed with policy formulation on TB diagnostics.

\section{Dynamic policy development on Xpert MTB/RIF}

In early September 2010, an Expert Group convened by WHO assessed the available data on Xpert MTB/ RIF, including information from six published papers [8-13], two large multicentre laboratory validation and demonstration studies coordinated by FIND [18, 19], results from cost-effectiveness analyses [17] and unpublished data from 12 investigator-driven, single-centre studies (most of which were subsequently published) shared with WHO under nondisclosure agreements. The GRADE evaluation assessed assay performance, the feasibility and anticipated impact of programmatic implementation, cost-effectiveness, and issues to be addressed in future research.

Recommendations from the Expert Group were subsequently endorsed by STAG-TB (Strategic and Technical Advisory Group for Tuberculosis) in late September 2010, and WHO was advised to proceed immediately with policy guidance, develop a global strategy for rapid update, convene a Global Consultation on implementation considerations, and assist countries with technical support and planning [20].

WHO convened a Global Consultation in early December 2010, which was attended by approximately 120 institutional and country representatives. An agreement was reached on interim diagnostic algorithms and the positioning of Xpert MTB/RIF in defined risk groups (MDR-TB and HIV-associated TB) at different levels of health services. Consensus agreements were incorporated into a subsequent WHO Rapid Implementation document [7] supported by an Xpert MTB/RIF checklist [21] providing practical suggestions for systematic roll-out of the assay to optimise use and benefits of the technology while addressing key operational research aspects in more longitudinal efforts. 

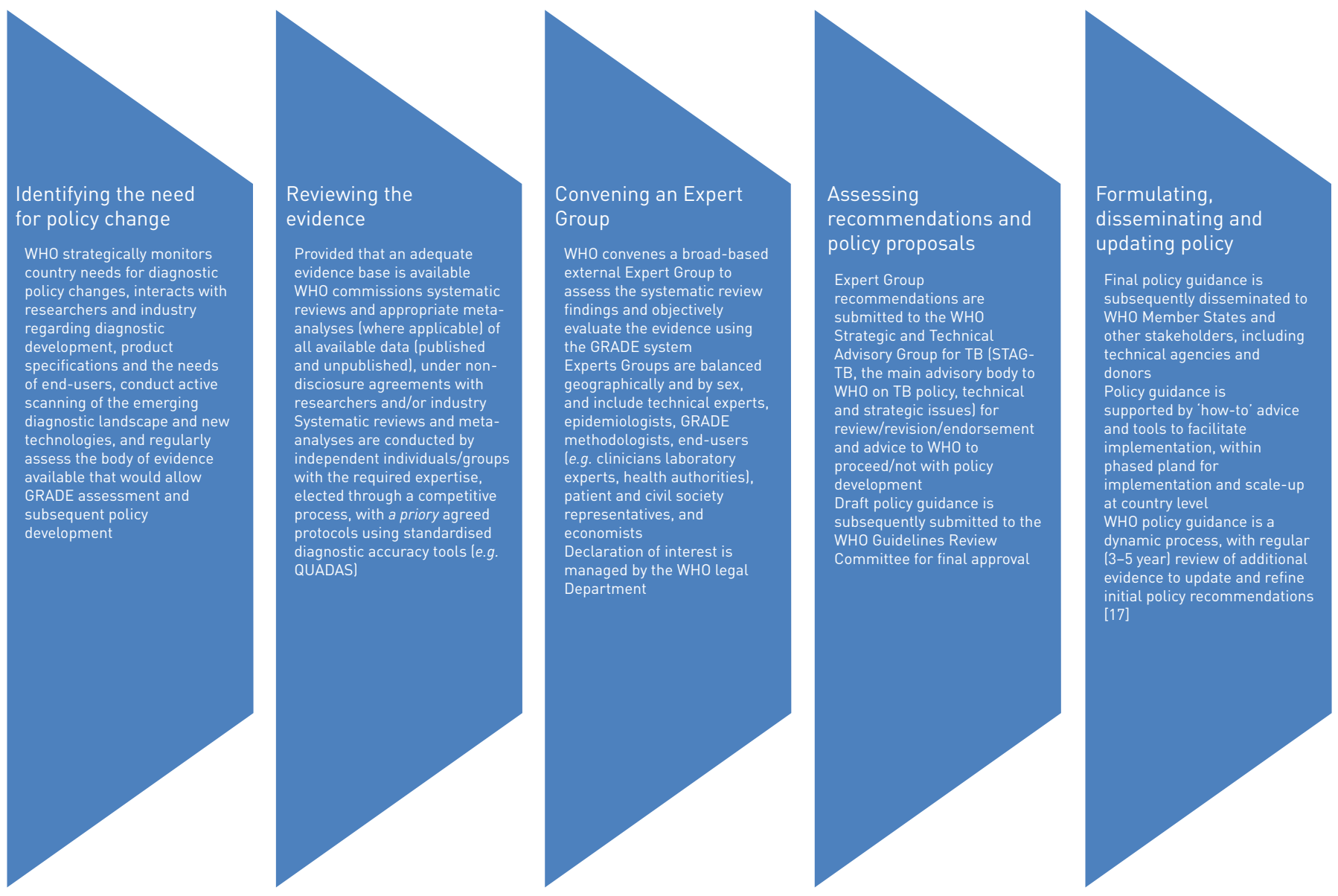

FIGURE 3 The World Health Organization (WHO) policy development process for tuberculosis (TB) diagnostics. GRADE: Grades of Recommendations Assessment, Development and Evaluation; QUADAS: Quality Assessment of Diagnostic Accuracy Studies; STAG-TB: Strategic and Technical Advisory Group for Tuberculosis.

In April 2011, WHO convened a meeting with early implementers of the Xpert MTB/RIF assay to refine the proposed diagnostic algorithms, develop a core set of variables to determine the impact of introducing the technology on laboratory workload, and clarify operational and logistical issues. A second WHO meeting of early implementers followed in April 2012 to share experiences during the introduction of the assay under routine TB control programme conditions.

Under its mandate to coordinate the global roll-out of Xpert MTB/RIF, WHO established a dedicated website and electronic data collection tool (http://who.int/tb/laboratory/mtbrifrollout/en/index.html), tracking country implementation and partner plans for scale-up in 145 countries eligible for preferential pricing of the assay, as well as to collect information from post-marketing surveillance of operational problems to guide scale-up of the technology under programmatic conditions.

Formal WHO policy recommendations on the use of Xpert MTB/RIF issued on December 8, 2010 arose from a solid GRADE assessment of the available evidence (table 2) [6].

\section{Analytical studies}

The Xpert MTB/RIF assay has analytic sensitivity of five genome copies of purified DNA, and $131 \mathrm{CFU} \cdot \mathrm{mL}^{-1}$ of $M$. tuberculosis spiked into sputum. No cross-reactivity with non-tuberculous mycobacteria (NTM) was detected. TB and resistance to rifampicin were correctly detected when NTM DNA or mixed susceptible and resistant strains were present. The sample reagent, added in a 2:1 ratio to sputum, killed $>6 \log _{10} \mathrm{CFU} \cdot \mathrm{mL}^{-1}$ of $M$. tuberculosis with $15 \mathrm{~min}$ of exposure and rendered $>97 \%$ of sputum smear-positive samples negative by Lowenstein-Jensen culture. No infectious aerosols were detected following the Xpert MTB/RIF inoculation procedure and sample testing [8-13]. 
TABLE 1 Evidence required by the World Health Organization (WHO) to enable tuberculosis (TB) diagnostic policy development and policy update

\section{Phase 1: research and development}

Typically consists of upstream research and development to define and validate a prototype, followed by laboratory validation under international standards that culminate in a design-locked product

WHO interacts with developers if requested to discuss end-user requirements such as biosafety, assay robustness and intended setting of use

\section{Phase 2: evaluation and demonstration}

The performance of the new diagnostic product should be evaluated in controlled trials at three to five trial sites in high-burden TB and HIV countries, ideally using pre-specified and-user product specifications. These data are often used for product registration with global and/or regulatory authorities such as FDA and/or CE marking.

Product specifications and performance should subsequently be validated in uncontrolled trials under field conditions in five to 10 trial sites in high-burden TB and HIV countries, and include cost-effectiveness studies.

\section{Phase 3: WHO evidence assessment using GRADE}

For new technologies or new indications for use of technologies already approved by WHO Submission of dossier with phase 1 and 2 data to WHO

Structured evidence assessment process (described in figure 3)

For fast-follower or generic versions of technologies already approved by WHO

Manufacture of the technology under ISO 13:485 standards; equivalent performance demonstrated in two to three independent supranational TB reference laboratories, to the reference technology already approved by WHO

Structured evidence assessment process (described in figure 3)

WHO is not a regulatory authority and does not recommend technologies for individual country use

\section{Phase 4: phased uptake and collection of evidence for scale-up}

New diagnostic successfully implemented in routine diagnostic services by early implementers in high-burden TB and HIV countries; systemic assessment of proposed algorithms, laboratory workload, operational constraints, country by cost-effectiveness. Lessons learnt by early implementers used for country adaptation.

Phase 5: scale-up and policy refinement

Scale-up of the new diagnostic, with subsequent data used to inform and refine WHO policy guidance in a dynamic and ongoing process

FDA: Food and Drug Administration; CE: Communauté Européenne (European Community); GRADE: Grades of Recommendations Assessment, Development and Evaluation; ISO: International Organization for Standardization.

\section{Controlled clinical validation trials}

The performance of Xpert MTB/RIF was tested in 1730 patients suspected to be affected by drug-susceptible or pulmonary MDR-TB from Peru, Azerbaijan, South Africa and India. Among culture-positive patients, a single, direct Xpert MTB/RIF test identified 98.2\% (551out of 561) of sputum smear-positive TB cases and $72.5 \%$ (124 out of 171) of those with sputum smear-negative TB. The test was specific in 604 (99.2\%) out of 609 patients not affected by TB. A second Xpert MTB/RIF test among patients with sputum smear-negative, culture-positive TB increased sensitivity by $12.6 \%$ and a third by $5.1 \%$, to reach $90.2 \%$. When compared to phenotypic DST, the Xpert MTB/RIF assay identified correctly 97.6\% (200 out of 205) of patients harbouring rifampicin-resistant strains and $98.1 \%$ (504 out of 514) of those with rifampicin-susceptible strains. Sequencing resolved all but two cases in favour of Xpert MTB/RIF [18].

\section{TABLE 2 World Health Organization (WHO) policy recommendations on Xpert MTB/RIF}

\section{The GRADE process confirmed a solid evidence base to support widespread use of Xpert MTB/RIF for detection of TB and rifampicin} resistance and resulted in the following main recommendations:

Xpert MTB/RIF should be used as the initial diagnostic test in individuals suspected of having MDR-TB or HIV-associated TB (strong recommendation)

Xpert MTB/RIF may be considered as a follow-on test to microscopy in settings where MDR-TB or HIV is of lesser concern, especially in further testing of smear-negative specimens (conditional recommendation, acknowledging major resource implications)

\section{Remarks}

These recommendations apply to the use of Xpert MTB/RIF in sputum specimens (including pellets from decontaminated specimens)

Data on the utility of Xpert MTB/RIF in extrapulmonary specimens are still limited

These recommendations support the use of one sputum specimen for diagnostic testing, acknowledging that multiple specimens increase the sensitivity of Xpert MTB/RIF but have major resource implications

These recommendations also apply to children, based on the generalisation of data from adults and acknowledging the limitations of microbiological diagnosis of TB (including MDR-TB) in children

Access to conventional microscopy, culture and DST is still needed for monitoring of therapy, prevalence surveys and/or surveillance and recovering isolates for DST other than rifampicin (including second-line anti-TB drugs)

GRADE: Grades of Recommendations Assessment, Development and Evaluation; MDR: multidrug resistant; DST: drug susceptibility testing. Data from [6]. 


\section{Field demonstration studies}

6648 individuals were prospectively enrolled in South Africa, Peru, India, Azerbaijan, the Philippines and Uganda, comparing Xpert MTB/RIF with microscopy in decentralised microscopy centres, and with culture and phenotypic DST in centralised laboratories. Xpert MTB/RIF detected 90.3\% (933 out of 1033) of the culture-confirmed TB cases, compared with $67.1 \%$ (699 out of 1041) using microscopy. In sputum smearnegative, culture-positive TB patients Xpert MTB/RIF test sensitivity was 76.9\% (296 out of 385) and specificity was $99.0 \%$ (2846 out of 2876). Sensitivity for rifampicin resistance was $94.4 \%$ (236 out of 250) and specificity was $98.3 \%$ (796 out of 810 ) [19].

While HIV co-infection substantially decreased the sensitivity of microscopy (to 47\%), Xpert MTB/RIF performance was not significantly affected. The median time to detection of TB was 0 days (interquartile range (IQR) 0-1) using Xpert MTB/RIF, compared to 1 day (IQR 0-1) for microscopy, 30 days (IQR 2343 ) for solid culture and 16 days (IQR 13-21) for liquid culture. The median time to detection of rifampicin resistance was 20 days (IQR 10-26) for line-probe assay versus 106 days (IQR 30-124) for phenotypic DST.

The Xpert MTB/RIF test reduced the median time to treatment for sputum smear-negative TB from 56 days (IQR 39-81) to 5 days (IQR 2-8). The indeterminate rate of Xpert MTB/RIF testing was 2.4\% (126 out of 5321 samples) compared to $4.6 \%$ (441 out of 9690) for culture.

\section{Unpublished, single-centre evaluation studies}

Results from 12 studies with varying design and study populations reported Xpert MTB/RIF sensitivity in detecting $\mathrm{TB}$ ranging from $70 \%$ to $100 \%$ in culture-positive patients and $\sim 60 \%$ in those with smearnegative disease. Specificity ranged from $91 \%$ to $100 \%$. Pooled average sensitivity for TB detection was $92.5 \%$ and pooled average specificity was $98 \%$. Average rifampicin sensitivity and specificity were $\sim 98 \%$ and $99 \%$, respectively.

\section{Operational and logistical issues}

The available evidence confirmed the robustness of the Xpert MTB/RIF assay under varying temperature and humidity conditions, the need for minimal staff training, basic biosafety requirements (as for sputum smear microscopy) and high levels of user satisfaction. Operational challenges included the requirement for an ambient temperature $<30^{\circ} \mathrm{C}$ (necessitating air conditioning in hot climates), and uninterrupted and stable electrical power supply (requiring generators in several sites). Storage space and conditions $\left(<28^{\circ} \mathrm{C}\right)$ for cartridges, waste generated (considerably more than for microscopy), and the 12-month shelf-life of cartridges were listed as main operational challenges $[6,7]$.

\section{Cost, affordability and cost-effectiveness analyses}

Using Xpert MTB/RIF for the diagnosis of smear-negative pulmonary TB was deemed cost-effective compared with existing diagnostic strategies in India, South Africa and Uganda, and within WHO acceptable incremental cost effectiveness ratios $[6,7,17]$.

The cost of achieving the diagnostic targets in the Global Plan to Stop TB, 2011-2015 [22] with and without use of Xpert MTB/RIF was appraised for three population groups, i.e. TB patients considered at risk of having MDR-TB, people living with HIV with TB signs and symptoms, and all people with TB signs and symptoms. Using the FIND negotiated price at the end of 2010 of US\$16.86 per cartridge, there were four main findings. First, a diagnostic strategy using Xpert MTB/RIF with follow-on DST for rifampicin-positive cases was a lower cost approach for reaching the 2015 targets for diagnosis of MDR-TB, both globally and in all high TB and high MDR-burden countries, compared with reliance on conventional culture and DST only. Secondly, using Xpert MTB/RIF to diagnose TB in people living with HIV in high HIV-prevalence settings was of similar or lower cost, compared with the conventional diagnostic algorithm (based on culture and radiography) recommended by WHO, in most countries. Thirdly, the total cost of using Xpert MTB/RIF to diagnose MDR-TB and TB among people living with HIV was a small fraction $(<5 \%)$ of total spending on TB control in 2010. Finally, the cost of using Xpert MTB/RIF to test all people with TB signs and symptoms was much higher compared with conventional diagnosis based on smear microscopy and radiographs, but in middle-income countries would be relatively affordable compared with total spending on TB care and control.

\section{Operational research informing policy on Xpert MTB/RIF}

Operational research on the Xpert MTB/RIF assay has proliferated subsequent to WHO endorsement. Of particular programmatic relevance are several operational research studies addressing key research questions identified following the WHO Global Consultation. As of July 2012, at least 24 operational research projects in 16 countries were registered, covering multiple implementation aspects [1]. 
By the end of August 2012, more than70 peer-reviewed publications, commentaries, viewpoints and editorials had been published [8-13, 17-19, 23-90], including an updated systematic review of 18 studies involving 10224 specimens, confirming the initial findings [23]. A single Xpert MTB/RIF test detected $90.4 \%$ of culture-confirmed pulmonary TB patients $(98.7 \%$ of smear-positive specimens and $75.0 \%$ of smear-negative specimens). Similar accuracy was retained in specimens from HIV-infected patients, showing pooled values of $81.7 \%$ sensitivity (95\% CI $77.0-85.8 \%$ ) and $98.0 \%$ specificity (95\% CI $96.6-$ 98.9\%). The accuracy of Xpert MTB/RIF in detecting paediatric TB was $74.3 \%$ (95\% CI $62.4-84.0 \%$ ). Accuracy in detecting extrapulmonary TB reached $70.7 \%$ sensitivity (95\% CI $59.6-80.3 \%$ ) and $82.6 \%$ specificity (95\% CI 79.5-85.3\%). Accuracy estimates for rifampicin resistance detection were equally similar to the initial data, with pooled sensitivity of $94.1 \%$ (95\% CI $91.6-96.0 \%)$ and pooled specificity of $97.0 \%$ (95\% CI 96.0-97.7\%).

Subsequent studies showed equally consistent results confirming the accuracy of the Xpert MTB/RIF assay in different settings and patient groups, with superior performance over microscopy. Most studies also confirmed the current operational limitations of the GeneXpert system: the sophisticated nature of the device requires care of handling, i.e. a stable and uninterrupted electrical supply to avoid interruption of the procedure and subsequent loss of results, an ambient temperature $<30^{\circ} \mathrm{C}$, security against theft, adequate storage space for the cartridges, and the need for sufficient staff to perform testing $[6,7,21]$.

As with any other TB test, the positive predictive value (PPV) of Xpert MTB/RIF testing is adversely affected in low disease-prevalence settings or populations.

\section{“Learning by doing": ongoing technological innovation and field experience}

As of June 2012, 67 low- and middle-income countries had implemented the Xpert MTB/RIF assay, with 749 GeneXpert machines, 3602 cartridge modules and 1.1 million cartridges deployed or used (fig. 4).

Initial anxiety about errors and invalid results was reported during implementation of Xpert MTB/RIF in selected countries $[24,65,70]$. Recurring errors at particular sites were linked to improper procedures in specimen collection and/or preparation of samples, and faulty modules and cartridges. Since the evaluation and demonstration studies published in 2011, refinements to the reagents and software of the Xpert MTB/ RIF assay have been made to decrease the frequency of false-positive rifampicin resistant results. Specifically, most of the false-positive results for rifampicin resistance were associated with a single probe; this has been redesigned to give more robust performance, with false-positive results for rifampicin resistance now rarely reported. The introduction of the latest generation Xpert MTB/RIF cartridge in December 2011 significantly reduced the number of signal loss (5011) errors, which had been the most commonly reported error [84].

The experience of the South African National Health Laboratory Service (NHLS) with over 300000 tests found a decreasing error rate in the presence of adequate training and troubleshooting, with a current overall error rate of $2.2 \%$ (W. Stevens, NHLS, Johannesburg, South Africa; personal communication), which is very similar to unreadable microscopy results and much lower than acceptable culture contamination rates.

Changes have been made to minimise packaging, thus reducing waste and shipping costs [84]. Real-time stability studies are being performed by FIND and Cepheid to increase the current cartridge shelf-life of 12 months to 24 months. Stability data for 18-month shelf-life determination are expected in May 2013 and for 24 months in November 2013 (M. Perkins, FIND; personal communication). An on-site calibration kit

FIGURE 4 Global uptake of Xpert MTB/RIF up to June 30, 2012. As of June 30, 2012, a total of 749 GeneXpert instruments (comprising 3602 modules) and 1107330 Xpert MTB/ RIF cartridges had been procured in the public sector in 67 of the 145 countries eligible for concessional pricing. Reproduced from [6] with permission from the publisher.

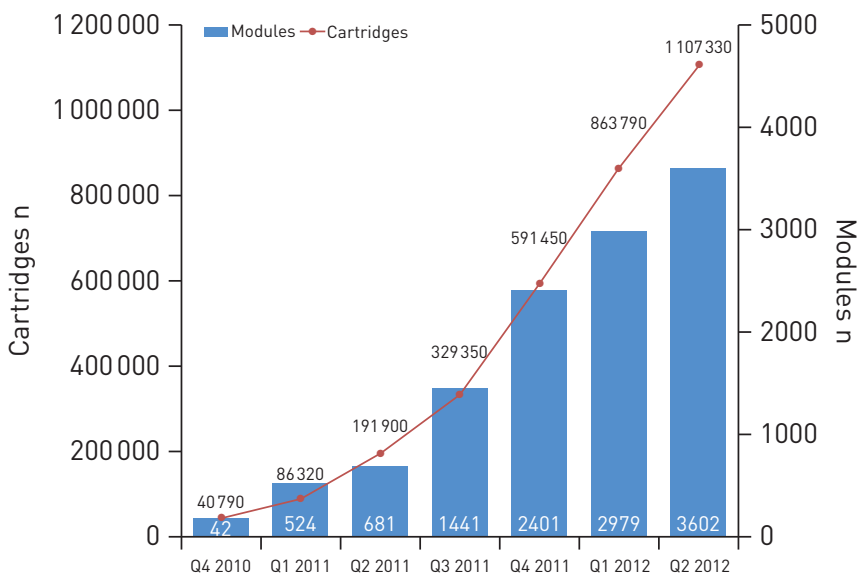


has been developed which allows users to recalibrate the optical system of the GeneXpert machine, verify the functioning thermal system, and conduct a series of system-level tests to ensure full system functionality within specifications, thus reducing the need for remote calibration of modules [84].

Experiences shared by early implementers showed that treatment of rifampicin-resistant TB cases diagnosed by Xpert MTB/RIF is a major, although controversial, concern [84]. While some argued for cautious rollout in order to ensure treatment access, others felt that diagnosis in the absence of appropriate treatment allows for increased advocacy for scale-up of treatment and enables patients to make appropriate life decisions and protect the health of their families, while also facilitating interventions for reduced transmission of drug-resistant strains in healthcare facilities.

Early implementers reported high levels of user acceptance and satisfaction [84], describing the technology as fast, easy-to-use, modern, and much less cumbersome than conventional TB diagnostic techniques. They also indicated that the time and resources needed to develop and implement effective diagnostic as well as clinical management algorithms should not be underestimated, and stressed the need for training of doctors and nurses on interpretation of Xpert MTB/RIF results and clinical management of patients [84].

Several early implementers felt that adoption of Xpert MTB/RIF by the large private sector in many highburden countries would be highly beneficial for increasing patient access to rapid and reliable diagnosis, while replacing poor technologies not endorsed by WHO [84]. Establishment of public-private collaborations was seen as mutually beneficial, allowing private providers to access concessional prices and national TB control programmes to ensure that patients detected in the private sector are duly reported and subsequently registered for appropriate treatment [84].

\section{Cost, affordability and cost-effectiveness}

While the Xpert MTB/RIF assay allows decentralised testing, cost and affordability of the assay are often cited as a barrier to wide-scale implementation. Price negotiations by FIND prior to launching the assay resulted in a significant upfront cost reduction (up to 85\%) and preferential pricing of both the GeneXpert instrument and Xpert MTB/RIF cartridges for the public sector in 145 low- and middle-income countries [7]. A further major price reduction in cartridge cost (from US\$16.86 to US\$9.98) was recently achieved following a novel financing agreement between the manufacturer and the Bill and Melinda Gates Foundation, the US Agency for International Development (USAID), the Office of the United States Global AIDS Coordinator (OGAC) and UNITAID [91]. Detailed analyses using the price of US\$9.98 per Xpert MTB/RIF cartridge to assess cost and affordability of diagnostic strategies confirmed and strengthened the findings of the analyses performed for the Global Consultation [85].

Studies of the cost and cost-effectiveness of Xpert MTB/RIF are currently limited to three countries: India, South Africa and Uganda [19, 46, 75-78, 85]. The data show that Xpert MTB/RIF is cost-effective compared with conventional diagnostic strategies, especially when the test is used as recommended by WHO, i.e. in persons suspected of MDR and/or HIV-associated TB. Use of the assay has also been found to be costeffective in pre-antiretroviral treatment (ART) screening and in reducing early mortality during the first 6 months of ART $[77,78]$. In South Africa, a diagnostic strategy of combining microscopy and Xpert MTB/ RIF was found to have produced the highest accuracy and lowest cost [17].

\section{Placement of Xpert MTB/RIF in tiered laboratory services}

Currently recommended TB diagnostics require different levels of laboratory sophistication due to technical complexity and biosafety concerns. To date, technologies to diagnose drug-resistant TB and smear-negative TB have been suitable for use only at the apex of tiered laboratory services, i.e. reference laboratories at central or regional level (table 3). A distinct advantage of Xpert MTB/RIF is its suitability for use at the district and sub-district level and the technology should therefore not be restricted to central/reference laboratory level only $[6,7]$.

Countries already using high-throughput liquid culture and DST systems or molecular line probe assay (LPA) for rapid diagnosis of rifampicin resistance at central/reference laboratory level should introduce Xpert MTB/RIF at lower health service levels. Selection of sites for placement of Xpert MTB/RIF testing should be guided by: 1) the prevalence of MDR or HIV-associated TB; 2) the current or estimated workload of the facility; 3) availability of adequate infrastructure; 4) availability of staff; and 5) availability and capacity for appropriate treatment $[6,7,21]$.

None of the existing TB diagnostic tools are mutually exclusive and implementation (in various combinations in country screening and diagnostic algorithms) is highly setting and resource specific [92]. One size no longer fits all, and expert laboratory input is needed to define the most cost-effective and 
TABLE 3 Summary of tuberculosis (TB) diagnostics evaluated by the World Health Organization 2007-2012

\section{7: Commercial liquid culture and DST systems}

Automated and manual commercial systems for liquid culture and DST recommended for use at central/reference laboratory level Phased implementation recommended within the context of comprehensive country plans for strengthening TB laboratory capacity

Currently regarded as the reference standard for conventional culture and DST and recommended as a stand-alone diagnostic test for TB and drug-resistance detection

\section{7: Rapid speciation strip technology}

Rapid chromatographic strip speciation recommended for distinguishing Mycobacterium tuberculosis from non-tuberculous mycobacteria

Recommended for use in combination with conventional culture and DST systems, at central/reference laboratory level

Recommended as a stand-alone speciation test for Mycobacterium tuberculosis isolates

2008: Molecular line probe assay for first-line anti-TB drugs

Commercial line probe assays recommended for rapid detection of rifampicin alone or in combination with isoniazid resistance detection in smear-positive sputum specimens and Mycobacterium tuberculosis isolates grown from culture, for use at central/reference laboratory level

Phased implementation recommended within the context of national plans for MDR-TB diagnosis, including development of country-specific screening/diagnostic algorithms

Can be used as a stand-alone diagnostic test for rifampicin resistance (but no other resistance) once laboratory proficiency and equivalence with commercial liquid culture systems have been validated

Need for conventional culture (for smear-negative sputum specimens and treatment monitoring) as well as phenotypic DST capacity remains 2010: LED microscopy

Recommended as immediate replacement for conventional fluorochrome microscopy and as gradual replacement for conventional light ZiehlNeelsen microscopy

Suitable for use at peripheral microscopy, as well as higher laboratory levels

2010: Selected non-commercial DST methods: MODS, NRA, CRI

Recommended as interim solutions for rapid rifampicin testing in resource-constrained settings, at central/reference laboratory level

Phased implementation under strict laboratory protocols and quality assurance recommended within the context of national plans for MDR-TB diagnosis, including development of country-specific screening/diagnostic algorithms

Phased implementation recommended within the context of national plans for MDR-TB diagnosis, including development of country-specific screening/diagnostic algorithms

Can be used as stand-alone diagnostic tests for rifampicin resistance (but no other resistance) once laboratory proficiency and equivalence with conventional culture systems have been validated

Need for conventional culture (for smear-negative sputum specimens and treatment monitoring) as well as DST capacity remains. MODS and NRA are suitable for direct testing on smear-positive sputum specimens and indirect testing on Mycobacterium tuberculosis isolates grown from culture

$\mathrm{CRI}$ is suitable for indirect testing on Mycobacterium tuberculosis isolates only

2011: Automated real-time nucleic acid amplification technology: Xpert MTB/RIF system

Recommended as rapid diagnostic test for TB and rifampicin resistance at peripheral microscopy, as well as higher laboratory levels

Can be used as stand-alone diagnostic test for TB detection in all settings (including HIV co-infected patients) and for rifampicin resistance in patients at risk of drug-resistant disease

Phased implementation and rapid scale-up recommended within the context of national TB and MDR-TB plans, including development of country-specific screening/diagnostic algorithms

Need for conventional microscopy and culture remains to monitor treatment and to conduct additional DST

Evaluated but not yet approved due to lack of adequate evidence

Sputum concentration and decontamination methods (evaluated 2008)

Phage-plaque technology for rapid rifampicin resistance detection (evaluated 2008)

Thin-layer agar methods for rapid culture and DST (evaluated 2010)

Molecular line probe assays for second-line anti-TB drugs (evaluated 2012)

Loop-mediated isothermal amplification test kit for tuberculosis (evaluated 2012)

Not approved for use

Commercial serodiagnostic tests for TB diagnosis (evaluated 2011)

IFN- $\gamma$ release assays for detection of active TB in all settings (evaluated 2011)

IFN- $\gamma$ release assays as replacement for TST to detect latent TB in low- and middle-income (typically high TB and/or HIV burden) settings (evaluated 2011)

DST: drug susceptibility testing; MDR: multidrug resistant; LED: light-emitting diode; MODS: microscopic observation of drug susceptibility; NRA: nitrate reductase assay; CRI: colorimetric redox indicator; IFN: interferon; TST: tuberculin skin test.

efficient algorithms in individual countries, guided by WHO standards and procedures, and within the context of overall, integrated laboratory strengthening activities [92].

\section{Targeting risk groups for testing}

Maximum benefit from the Xpert MTB/RIF assay is obtained by targeted testing of individuals considered at risk of drug-resistant TB and/or smear-negative TB, such as those co-infected with HIV. Risk groups for drug-resistant TB include all re-treatment categories (i.e. failure, relapse and default cases), as well as those 
described in WHO guidelines [93, 94] or national policies, including those with HIV infection [95]. These individuals should receive an Xpert MTB/RIF test as a primary diagnostic test, i.e. subsequent confirmation of the diagnosis is not required and appropriate treatment should be started on the basis of the Xpert MTB/ RIF result.

Published studies have shown significant increases in TB case detection when Xpert MTB/RIF is used as an add-on or replacement test for microscopy, especially in settings with high HIV prevalence [18, 19, 34, 35, $50,61]$. Both diagnostic delay and treatment initiation can be significantly shortened compared to conventional approaches [92], reducing premature death and on-going transmission. The 2012 WHO Global Report [1] also notes the still insufficient screening of HIV patients for TB and the low proportion of patients started on isoniazid preventive therapy.

HIV testing should be routinely offered to all persons suspected of having TB, based on WHO recommendations [95], ideally before investigation with Xpert MTB/RIF. Up to 25\% of patients accessing HIV services may have active $\mathrm{TB}$, the vast majority of which would be missed using conventional microscopy as a primary diagnostic tool [96]. The systematic introduction of Xpert MTB/RIF in HIV services would, therefore, make a major contribution to intensified TB case finding efforts and increased uptake of isoniazid preventive therapy.

The distinct advantage of Xpert MTB/RIF in providing a rapid, simultaneous diagnosis of both TB and rifampicin resistance has also given rise to continuing debate and concerns about the implications of positive results in different epidemiological and resource settings [24, 42, 43, 79, 80]. It is therefore important to distinguish the performance characteristics and treatment implications of the assay for: 1) TB detection and; 2) rifampicin resistance detection.

In many settings, the vast majority of persons suspected of having TB will not have risk factors for drug resistance or be HIV-positive. Careful consideration should be given in these circumstances to the resource implications of offering routine Xpert MTB/RIF testing $[6,7]$ and the low PPV of the assay for detecting rifampicin resistance at a low underlying prevalence (tables 4 and 5). Where resources are limited, national $\mathrm{TB}$ control programmes will have to prioritise specific groups for testing, decide whether Xpert MTB/RIF is performed as an initial diagnostic test or as a follow-on test after sputum smear microscopy, and consider the use of chest radiography as a first screening tool.

\section{Predictive values of Xpert MTB/RIF for TB case detection}

In the GRADE framework, diagnostic test accuracy can be interpreted as proxy measures for patientimportant outcomes based on the relative importance/impact of false-positive and false-negative results [16]. Poor sensitivity would result in false-negative results with adverse consequences for patient morbidity and mortality and ongoing disease transmission. Poor specificity would result in false-positive results exposing patients to unnecessary treatment while the underlying cause of disease remains undiagnosed.

Test accuracy is also dependent on underlying disease prevalence. Typically, between $10 \%$ and $20 \%$ of persons with respiratory symptoms may have confirmed TB in high-burden settings. Table 4 presents the predictive values for TB detection using Xpert MTB/RIF (compared to conventional culture) in settings or populations with varying TB prevalence. The negative predictive value (NPV) is over $99 \%$ in settings with both low and high prevalence of TB, i.e. a negative result reliably excludes TB. Table 4 shows that the vast majority of patients with a negative Xpert MTB/RIF result in such settings will not have TB and very few false-positive results will occur. Even with a low PPV the absolute number of false-positives will usually be very low and the proportion of overall true results (positive and negative combined) far outweigh the proportion of overall false results.

\section{Predictive values of Xpert MTB/RIF for rifampicin resistance detection}

Table 5 presents PPV and NPV for rifampicin resistance detection using Xpert MTB/RIF in settings or populations with varying prevalence of rifampicin resistance. The NPV is over $99 \%$ in settings with both low and high prevalence of rifampicin resistance, i.e. a negative result reliably excludes resistance and no further testing to confirm negative results is required.

The PPV for rifampicin resistance using Xpert MTB/RIF exceeds 90\% in settings or patient groups where the underlying prevalence of rifampicin resistance is $>15 \%$ (table 5). In settings or patient groups where rifampicin resistance is rare, the PPV of Xpert MTB/RIF (and any other test) is adversely affected, significantly diminishing when rifampicin resistance prevalence falls below $5 \%$.

The PPV for rifampicin resistance using Xpert MTB/RIF (or any other test) can be substantially improved by careful risk assessment in individual patients and targeted testing of risk groups: drug resistance surveillance data from 114 countries showed that the weighted proportion of MDR among previously 
TABLE 4 False positive, false negative and predictive values for tuberculosis (TB) detection using Xpert MTB/RIF

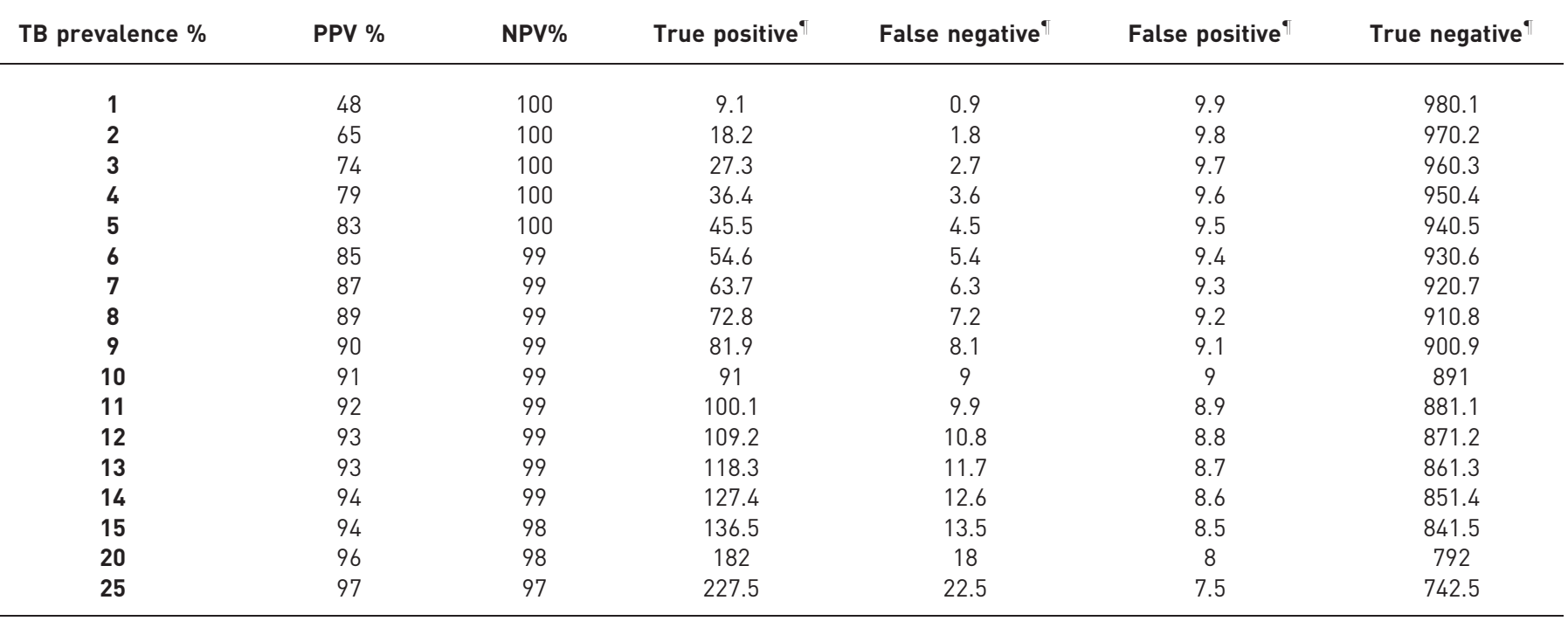

PPV: positive predictive value; NPV: negative predictive value. \# : according to varying TB prevalences in a sample population of 1000 individuals; ": sensitivity (91\%) and specificity (99\%) for Xpert MTB/RIF TB detection, compared with reference method (culture).

treated cases is $19.8 \%$ (95\% CI 14.4-25.1), which is several times higher than the proportion of new TB cases with MDR (3.4\%; 95\% CI 1.9-5.0) [3]. Therefore, even in low MDR-TB prevalence settings, testing previously treated patients should result in high PPV for rifampicin resistance, allowing treatment to be initiated based on the Xpert MTB/RIF result. Testing new TB cases not at risk of MDR-TB in low MDR-TB prevalence settings will, however, result in low PPV, requiring confirmation of rifampicin resistance by phenotypic DST or LPA (and not by a second Xpert MTB/RIF test) prior to treatment initiation.

The performance of Xpert MTB/RIF has been evaluated against existing reference standards, i.e. microscopy and culture for TB testing and phenotypic DST for rifampicin resistance testing. None of the currently available microbiological reference methods is $100 \%$ accurate, a well-recognised constraint in TB diagnostic test development and evaluation. Emerging data seem to suggest that low-level but potentially clinically relevant rifampicin resistance linked to infrequent $r p o B$ mutations may be missed by standard growth-based

TABLE 5 False positive, false negative and predictive values for rifampicin resistance using Xpert MTB/RIF

\begin{tabular}{|c|c|c|c|c|c|c|}
\hline Rifampicin resistance prevalence \% & PPV \% & NPV \% & True positive & False negative & False positive & True negative \\
\hline 1 & 32.4 & 99.9 & 9.5 & 0.5 & 19.8 & 970.2 \\
\hline 2 & 49.2 & 99.9 & 19 & 1 & 19.6 & 960.4 \\
\hline 4 & 66.4 & 99.8 & 38 & 2 & 19.2 & 940.8 \\
\hline 5 & 71.4 & 99.7 & 47.5 & 2.5 & 19 & 931 \\
\hline 6 & 75.2 & 99.7 & 57 & 3 & 18.8 & 921.2 \\
\hline 9 & 82.4 & 99.5 & 85.5 & 4.5 & 18.2 & 891.8 \\
\hline 10 & 84.1 & 99.4 & 95 & 5 & 18 & 882 \\
\hline 11 & 85.4 & 99.4 & 104.5 & 5.5 & 17.8 & 872.2 \\
\hline 12 & 86.6 & 99.3 & 114 & 6 & 17.6 & 862.4 \\
\hline 13 & 87.7 & 99.2 & 123.5 & 6.5 & 17.4 & 852.6 \\
\hline 14 & 88.5 & 99.2 & 133 & 7 & 17.2 & 842.8 \\
\hline
\end{tabular}

PPV: positive predictive value; NPV: negative predictive value. " : according to varying prevalences of rifampicin resistance in a sample population of 1000 individuals; " : sensitivity (95\%) and specificity (98\%) for Xpert MTB/RIF rifampicin resistance, compared with reference method (culture). 
methods, particularly the automated broth-based systems [97]. Sequencing, albeit limited, has largely resolved discordant results in favour of Xpert MTB/RIF, although a few truly false-positive results have been reported [17, 18, 84]. Additional data on mutation sequencing of $M$. tuberculosis strains and the clinical outcomes of patients with rifampicin resistance detected by Xpert MTB/RIF are therefore highly desirable.

\section{Use of Xpert MTB/RIF in diagnosis of paediatric TB}

Laboratory diagnosis of TB in children remains a real challenge due to the low sensitivity of sputum smear microscopy, the difficulty in collecting sufficient and high-quality specimens, and a substantial proportion of paediatric cases with extrapulmonary involvement.

Current WHO policy recommendations on the use of Xpert MTB/RIF in children are extrapolated from data on adults $[6,7]$, given the well-known limitations of microbiological methods in diagnosing paediatric TB. Subsequent studies have shown a significant improvement in diagnosing TB in children using the Xpert MTB/RIF assay.

Both high sensitivity (86.9\%) and specificity (99.7\%) of Xpert MTB/RIF in extrapulmonary paediatric samples ( $\mathrm{n}=344$, mainly gastric aspirates and biopsies) were reported by TORTOLI et al. [72], using positive culture and/or therapeutic response as a composite standard. In a large study on young South African children (including 24\% with HIV co-infection), NICOL et al. [50] showed a sensitivity of $74.3 \%$. Using Xpert MTB/RIF on two induced sputum specimens detected twice as many cases $(75.9 \%)$ compared to sputum smear $(38 \%)$ resulting in an overall Xpert specificity of $98.8 \%$. Similar results were obtained by RACHOw et al. [71] using Xpert MTB/RIF for diagnosis of pulmonary TB in $164(51.2 \%)$ older children in a high HIV prevalence setting.

\section{Use of Xpert MTB/RIF in diagnosis of extrapulmonary TB}

The diagnosis of extrapulmonary TB poses a serious challenge due to the pleomorphic presentation of the disease. Samples collected for microbiological diagnosis are often paucibacillary, resulting in a low sensitivity of smear microscopy and earlier nucleic acid amplification tests.

Several studies have now assessed the performance of Xpert MTB/RIF in the diagnosis of extrapulmonary TB $[23,27-31,38,39,53-56,59-62]$. The sensitivity and specificity ranged between $77 \%$ and $95 \%$ for biopsy, urine and pus while it was lower than $50 \%$ for cavitary fluids [23]. The specificity in these specimens ranged from $97 \%$ to $100 \%[23]$.

\section{Management of patients detected by Xpert MTB/RIF}

TB patients identified by Xpert MTB/RIF without rifampicin resistance should receive appropriate first-line anti-TB treatment immediately. HIV co-infected patients detected by Xpert MTB/RIF should be managed according to WHO guidelines, including HIV clinical staging, immunological staging with CD4 count, initiation of co-trimoxazole preventive therapy and initiation of antiretroviral therapy irrespective of CD4 count [95].

Rapid DST for rifampicin is recommended by WHO [92-94]. Patients at risk of drug resistance in whom rifampicin resistance is detected by Xpert MTB/RIF should be placed on an appropriate MDR-TB regimen immediately and isoniazid added until the DST result for isoniazid is available. These patients should provide an additional sputum specimen for conventional culture and DST against other first- and secondline drugs according to WHO recommendations [93, 94], and their treatment adjusted accordingly.

Molecular tests, including Xpert MTB/RIF, are not suitable for patient monitoring as these tests detect DNA from both viable and non-viable bacilli. Conventional laboratory capacity is, therefore, required to monitor treatment response of patients detected by Xpert MTB/RIF and to conduct additional DST in patients with rifampicin resistance.

Patients whose diagnosis of TB is confirmed by Xpert MTB/RIF and who have rifampicin-susceptible TB disease should be monitored during treatment with sputum smear microscopy. No additional sputum smear microscopy examination needs to be performed for establishing baseline status. Sputum smear microscopy should be performed at completion of the intensive phase of treatment, 5 months into treatment and at the end of treatment as per WHO guidelines [98].

Treatment outcomes for patients with a positive smear culture or Xpert MTB/RIF result at the start of treatment should be categorised according to current WHO guidelines [7, 98]. Current treatment outcome definitions apply, including the outcome "cured", i.e. a patient with a positive Xpert MTB/RIF test (only) at baseline can be declared cured if negative smear results during and at the end of the treatment were recorded. 
Patients placed on MDR-TB treatment should be monitored by sputum culture as per current WHO guidelines. If resources permit, monthly culture throughout treatment is recommended given that this has shown the highest benefit to detect failures [99].

\section{Use of Xpert MTB/RIF vis à vis other tests}

From a purely technical perspective, no test for TB is perfect. Xpert MTB/RIF limitations have been described previously. Microscopy, conventional culture and DST (both phenotypic and genotypic) all have shortcomings and limitations related to accuracy and effectiveness, operator dependency, training and resource requirements, and biosafety. WHO policy guidance on new TB diagnostics takes all these aspects into careful consideration [92], balancing test accuracy with potential harms and benefits, operational considerations, resource implications and anticipated public health impact.

As outlined previously, Xpert MTB/RIF efficiency is maximised and cost minimised by targeted testing of individuals at risk of drug resistance and/or HIV co-infection. In these patient groups, Xpert MTB/RIF clearly outperforms microscopy and should be used as the initial diagnostic test.

Xpert MTB/RIF is currently the only DST technology suitable beyond central/reference laboratory level and should be the first point of testing when MDR-TB is suspected. It is a relatively low throughput technology (maximum of 20 specimens per day in the four-module GeneXpert machine). Settings with higher patient loads should consider bigger capacity machines or the referral of specimens to central/national laboratory levels for first-line LPA or phenotypic DST (both high through-put technologies).

All DST methods currently recommended by WHO show similar accuracy for rifampicin resistance detection. All tests have poor PPV in settings with low levels of MDR-TB and good PPV in settings with high levels. In settings where rifampicin/MDR resistance is rare, resistance by any test therefore needs to be confirmed by an alternative WHO-recommended DST method. Specimens from confirmed MDR-TB patients need to undergo phenotypic DST against fluoroquinolones and kanamycin, amikacin and capreomycin to check for extensively drug-resistant-TB [93, 94].

Strategies for Xpert MTB/RIF testing of all persons suspected of having TB will be strongly dependent on available resources and the screening and diagnostic algorithms at country level. TB screening as per national guidelines should take place and pre-test screening strategies, including chest radiography, should be considered to optimise Xpert MTB/RIF efficiency and cost. Individuals with sputum smear-positive microscopy results do not need to be retested with Xpert MTB/RIF unless they belong to the risk groups for drug resistance, as described previously.

In summary, Xpert MTB/RIF does not eliminate the need for traditional bacteriological methods (direct examination, culture and DST) and for other rapid molecular methods. National programmes need to develop setting-specific, evidence-based and cost-effective algorithms designed to ensure universal access to quality diagnosis for all TB cases.

\section{Use of Xpert MTB/RIF in prevalence surveys and drug resistance surveillance}

Upgrading laboratory infrastructure and strengthening capacity for culture and DST are among the most important indirect benefits of implementing a drug resistance survey [100] as many laboratories need considerable refurbishment and/or upgrade, training of staff, and procurement of equipment and consumables before starting a survey [101]. Although not a complete surrogate for MDR-TB, particularly in settings with low resistance levels [102], rifampicin resistance is the most important indicator of MDR-TB, with serious clinical implications for affected patients.

At least two groups of countries could benefit considerably from the use of Xpert MTB/RIF as a screening tool in drug resistance surveys. The first group is countries in which laboratories would struggle to cope with the huge workload generated by a survey while managing their routine work and maintaining highquality standards. The second group is countries where there is no capacity to perform culture and DST. In these settings, instead of relying entirely on testing abroad, usually at a TB Supranational Reference Laboratory, with increased logistics and operational costs, Xpert MTB/RIF could be used to screen specimens and identify those requiring further testing in a specialised laboratory.

Given that most patients enrolled in drug resistance surveys are newly diagnosed with TB and at low risk of rifampicin resistance, the PPV of any test will not be adequate to identify true positives but the NPV will be sufficiently high to accurately identify true negatives. Xpert MTB/RIF could, therefore, be used as screening tool to identify those with no resistance to rifampicin, while patients with rifampicin resistance undergo further confirmatory testing with a second WHO-approved technology. 
In a recent TB prevalence study, Dorman et al. [86] suggested the potential use of the Xpert MTB/RIF as a single testing strategy: the diagnostic yield of M. tuberculosis was 2.7\% (187 out of 6893) for liquid culture, $2.1 \%$ (144 out of 6893) for Xpert MTB/RIF and 1.3\% (91 out of 6893) for smear microscopy. Agreement of Xpert MTB/RIF with liquid culture was 98.5\% (95\% CI 98.2-98.8\%) and respective test failure rates (noninterpretable results) were $0.3 \%$ for Xpert MTB/RIF and $3.6 \%$ for liquid culture. Overall Xpert MTB/ RIF sensitivity was 62.6\% (95\% CI 55.2-69.5\%), specificity was 99.6\% (95\% CI 99.4-99.7\%), PPV was 81.3\% (95\% CI 3.9-87.3\%), and NPV was 98.9\% (95\% CI 98.6-99.2\%) [86]. While these results are encouraging, more evidence is needed on the use of Xpert MTB/RIF in the context of prevalence surveys and other case-finding strategies in which $\mathrm{TB}$ prevalence and the pre-test probability of TB disease are relatively low.

\section{Aligning diagnostic and treatment capacity}

Lack of diagnostic capacity has been a longstanding and major barrier to scaling up MDR-TB care. The advent of new TB diagnostics, and Xpert MTB/RIF in particular, allows this constraint to be largely overcome. Early implementers have reported a $30-40 \%$ increase in the number of drug-susceptible TB patients being detected after roll-out of Xpert MTB/RIF, while MDR-TB cases have increased two- to threefold in many settings $[17,18,71]$.

Providing a definitive diagnosis for the large proportion of drug susceptible TB cases currently being reported without laboratory confirmation would allow treatment to be shifted away from those who do not need it to those who do. For the $\geqslant 80 \%$ of MDR-TB patients estimated to arise each year but remaining undiagnosed, prompt and appropriate treatment would prevent premature death, reduce the risk for aggravating drug resistance, and curtail disease transmission. Introduction of Xpert MTB/RIF should also allow for more robust and reliable forecasting of patient numbers, one of the most pressing constraints in securing adequate availability of second-line drugs. This in turn could stimulate more investment into second-line drugs and drive down the exorbitant cost of MDR-TB treatment.

An unintended consequence of scaling up diagnostics is the risk that patients diagnosed with MDR-TB cannot access the complex, second-line drug treatment required. This raises the question of ethics, human rights and public health. Should a diagnosis not lead to appropriate treatment? Concerns about the ethics of rolling out Xpert MTB/RIF in developing countries in the presumed absence of treatment for MDR-TB have been raised $[42,87]$, as have the counterpoint on the ethics of not rolling out the assay in low-income countries[43, 88].

Public health, ethics and human rights should be balanced when addressing the cost, risks and benefits, and technical limitations of any new, transformational intervention. Systematic roll-out of the Xpert MTB/RIF assay complies fully with WHO guidance on ethics of TB prevention, care and control [103], as well as the WHO-endorsed "progressive realisation" approach which states that "while countries are in the process of scaling up treatment, the use of DST can be appropriate as an interim measure even when no second-line drug treatment is available, or when the only available treatment is substandard"[43]. Virtually all lowincome countries have ratified the International Covenant on Economic, Social, and Cultural Rights (ICESR) governing the WHO-endorsed strategy of progressive realisation. We therefore agree with leading global ethicists that public health, ethics and human rights obligations apply equally to high TB burden lowincome countries as they do to resource-rich countries and that the public health potential of the Xpert $\mathrm{MTB} / \mathrm{RIF}$ assay should be considered despite cost and operational considerations $[43,103]$.

\section{Research needs}

As countries start to implement Xpert MTB/RIF they will be facing operational and logistical challenges related to changes in screening and diagnostic algorithms, shifts in laboratory organisation and workload, and requirements for improved supply chain management. In addition, country-specific adaptation of the diagnostic algorithms (e.g. prioritisation of patient groups to be tested) may be dictated by the availability of resources. WHO has therefore recommended that roll-out of Xpert MTB/RIF be addressed in a systematic and coordinated approach to optimise the usefulness of the technology under routine programme conditions and to ensure maximum efficiency $[6,7]$. In addition, WHO has recommended ongoing operational research to refine and inform future policy, in line with the requirement for dynamic policy guidance by the WHO Guidelines Review Committee [16].

More studies merely evaluating the performance of the Xpert MTB/RIF assay against conventional diagnostics in detecting pulmonary TB are not expected to challenge or change the existing evidence base. The assay has been shown repeatedly to be highly accurate, particularly if used in targeted testing as recommended by WHO. Implementation research should therefore now focus on the "how" and "when" of Xpert MTB/RIF implementation and scale-up, informed by appropriately designed studies (and using real 
data) that evaluate the test's impact and cost-effectiveness when used in different algorithms and with other screening and diagnostic tests. Policy refinement will also benefit from additional data on the use of the assay in extrapulmonary and paediatric $\mathrm{TB}$, in prevalence surveys and drug resistance surveillance, and in active case finding.

Xpert MTB/RIF MTB/RIF roll-out can, and should, serve as a pathfinder for implementation of future TB tests by providing national TB control programmes with data to develop long-term TB diagnostic strategies. Experiences and lessons learnt from programmatic roll-out (i.e. evidence for scaling up) will inform and facilitate eventual country-wide scale-up and assist other countries intending to embark on the same process.

Cost and potential disruption of health services are characteristic consequences of introducing any new public health intervention tool [88]. Rather than blocking or slowing down the introduction of new technologies [87] or waiting until ideal operational conditions are in place [89], scientific debate should focus on whether patient and public health benefits warrant implementation, even of a so-called "disruptive" intervention. Although not expected to show overall cost disadvantages, in-depth, cost-effectiveness studies on the impact of Xpert MTB/RIF in different settings would therefore be advantageous, especially since the assay will be used in varied diagnostic algorithms and underlying TB and MDR-TB epidemiology.

On a more fundamental research level, second-generation Xpert MTB/RIF tests with probes to detection resistance other than rifampicin will be most advantageous. In addition, the development of competing technologies with comparable performance and ease-of-use to the Xpert MTB/RIF assay is strongly encouraged to generate increased demand and market competition. Most pressing is the need for a robust, low-cost and safe point-of-care diagnostic for $\mathrm{TB}$ and drug-resistant $\mathrm{TB}$. This will require dramatic increases in research investment to identify appropriate biomarkers and capitalise on technological breakthroughs to create innovative test platforms [90]. The experiences in HIV test and drug development have shown the advantages gained from innovation and solid investment in research [104], strikingly different from the TB research world that remains woefully under-funded [105].

A summary of the current evidence available on Xpert MTB/RIF [8-13, 18, 19, 27-41, 50-74, 86, 106-112] is available in the supplementary material.

\section{Conclusion}

In the mid-1990s, when WHO declared TB a global emergency and subsequently introduced the DOTS (directly observed treatment, short course) strategy, the impact of the HIV epidemic on the dynamics of TB control (especially in Africa) was not fully realised, and no information on the public health impact of the growing problem of TB drug resistance was available. Under the assumption that MDR-TB was a rare event, good microscopy services were deemed sufficient to control TB in most settings. Indeed, many national programmes witnessed annual decreases in TB case rates following the wide implementation of microscopy services linked to the use of short-course chemotherapy under close supervision. Before the end of the 20th century, however, three events suggested that microscopy would become inadequate. The first was the magnitude of the HIV pandemic and its extraordinary impact on susceptibility to TB. The second was the growing burden and geographical spread of MDR-TB. Thirdly was the very slow decline in TB incidence in countries implementing DOTS, even when the prevalence of MDR-TB and HIV co-infection was low.

In 2006, WHO introduced the Stop TB Strategy which, in addition to the essential elements of the DOTS strategy, included measures specifically targeting (amongst others) proper care of HIV-associated TB and MDR-TB. As a result, in 2009, the World Health Assembly called for universal access to culture and DST, marking a dramatic shift in strategy. The updated Global Plan to Stop TB, 2011-2015 [22], underpinned by the Stop TB Strategy, called for massive investment in laboratory services to achieve screening for MDR in at least $20 \%$ of new TB cases and $100 \%$ of those previously treated by 2015 . It also called for $>50 \%$ of all smear-negative cases to be tested with molecular or culture-based methods. Data reported to WHO in 2012 clearly show that these targets are not on track. Reasons include huge gaps in funding to establish the required laboratory infrastructure, slow diagnostic policy reform at country level, and critical shortages of specialised laboratory staff.

WHO policy guidance on Xpert MTB/RIF recognises its potential in addressing some of the most pressing barriers to rapid diagnosis of $\mathrm{TB}$ and drug-resistant $\mathrm{TB}$, and has attempted to provide the necessary information and support to enable countries to make appropriate decisions on its utilisation. The WHO guidance also explicitly highlights the resource implications of rolling out the technology, as well as the need to ensure appropriate treatment of those patients detected; however, increased demand for testing, the difficulties in providing care for drug-resistant patients, and concerns about affordability should not be the prime drivers delaying roll-out of new and innovative interventions. 
Evidence on "where" to locate Xpert MTB/RIF (peripheral versus central laboratories) and "whom" to test (targeted versus general use) is growing, allowing rational and sustainable roll-out of the technology even in resource-constrained settings.

The experience of HIV testing despite inadequate treatment facilities provides a solid precedent for TB to follow: in the HIV world, moral pressure has been put on drug and diagnostics manufacturers to lower the prices of their products and to develop novel ones. Increased demand for drugs as a result of improved case detection has created scale and ultimately lowered prices, thus facilitating increased access.

As countries adjust their diagnostic algorithms to accommodate Xpert MTB/RIF roll-out, diagnostic paradigms for HIV-associated and drug-resistant TB are expected to shift significantly, away from highly centralised, complex diagnostic algorithms and referral systems (with inevitable long delays) towards simplified diagnostic approaches for at-risk patients at decentralised levels of the health system. These should be accompanied by more focused use of screening methods to increase the pre-test probability of TB prior to Xpert MTB/RIF testing, accelerated implementation of WHO screening policies for TB-HIV using Xpert MTB/RIF as the initial diagnostic test, and more focused identification of patients suspected of having to suffer from MDR-TB, using Xpert MTB/RIF as a rapid test rather than waiting for patients to fail firstline therapy before proceeding with culture and DST.

Stagnation in TB control and MDR-TB care delivery has severe consequences for TB patients, who often belong to the most vulnerable and neglected sector of society. Scientific breakthroughs such as the Xpert MTB/RIF assay (and hopefully additional new diagnostics, drugs and vaccines coming to use in the next few years) should not be withheld from these marginalised groups but deployed without undue delay, optimising patient and public health benefits.

\section{References}

World Health Organization. Global Tuberculosis Control Report. Geneva, World Health Organization, 2012.

World Health Organization. Towards Universal Access to Diagnosis and Treatment of Multidrug-resistant and Extensively Drug-resistant Tuberculosis by 2015. WHO progress report. Geneva, World Health Organization, 2011. Zignol M, van Gemert W, Falzon D, et al. Surveillance of anti-tuberculosis drug resistance in the world: an updated analysis, 2007-2010. Bull World Health Organ 2012; 90: 111-119D.

4 Matteelli A, Centis R, D'Ambrosio L, et al. Multidrug-resistant tuberculosis today. Bull World Health Organ 2012; 90: 78.

5 Raviglione M, Marais B, Floyd K, et al. Scaling up interventions to achieve global tuberculosis control: progress and new developments. Lancet 2012; 379: 1902-1913.

6 World Health Organization. Policy Statement: Automated real-time Nucleic Acid Amplification Technology for Rapid and Simultaneous Detection of Tuberculosis and Rifampicin Resistance: Xpert MTB/RIF System. Geneva, World Health Organization, 2011.

7 World Health Organization. Rapid Implementation of the Xpert MTB/RIF Diagnostic Test. Technical and Operational "How-to" Practical Considerations. Geneva, World Health Organization, 2011.

8 Piatek AS, Tyagi S, Pol AC, et al. Molecular beacon sequence analysis for detecting drug resistance in Mycobacterium tuberculosis. Nat Biotechnol 1998; 16: 359-363.

9 Piatek AS, Telenti A, Murray MR, et al. Genotypic analysis of Mycobacterium tuberculosis in two distinct populations using molecular beacons: implications for rapid susceptibility testing. Antimicrob Agents Chemother 2000; 44: 103-110.

10 El-Hajj HH, Marras SAE, Tyagi S, et al. Detection of rifampin resistance in Mycobacterium tuberculosis in a single tube with molecular beacons. J Clin Microbiol 2001; 39: 4131-4137.

11 Helb D, Jones M, Story E, et al. Rapid detection of Mycobacterium tuberculosis and rifampin resistance by use of ondemand, near-patient technology. J Clin Microbiol 2010; 48: 229-237.

12 Blakemore R, Story E, Helb D, et al. Evaluation of the analytical performance of the Xpert MTB/RIF assay. J Clin Microbiol 2010; 48: 2495-2501.

13 Banada P, Sivasubramani SK, Blakemore R, et al. Containment of bioaerosol infection risk by the Xpert MTB/RIF assay and its applicability to point-of-care settings. J Clin Microbiol 2010; 48: 3551-3557.

14 Schünemann HJ, Oxman AD, Brozek J, et al. Grading quality of evidence and strength of recommendations for diagnostic tests and strategies. BMJ 2008; 336: 1106-1110.

15 World Health Organization. Handbook for Guideline Development. Geneva, World Health Organisation, 2012.

16 Hsu J, Brozek JL, Terraciano L, et al. Application of GRADE: making evidence-based recommendations about diagnostic tests in clinical practice guidelines. Implement Sci 2011; 6: 62.

17 Vassall A, van Kampen S, Sohn H, et al. Rapid diagnosis of tuberculosis with the Xpert MTB/RIF assay in high burden countries: a cost-effectiveness analysis. PLoS Med 2011; 8: e1001120.

18 Boehme CC, Nabeta P, Hillemann D, et al. Rapid molecular detection of tuberculosis and rifampin resistance. N Engl J Med 2010; 363: 1005-1015.

19 Boehme CC, Nicol MP, Nabeta P, et al. Feasibility, diagnostic accuracy, and effectiveness of decentralised use of the Xpert MTB/RIF test for diagnosis of tuberculosis and multidrug resistance: a multicentre implementation study. Lancet 2011; 377: 1495-1505.

20 World Health Organization. Strategic and Technical Advisory Group for Tuberculosis (STAG-TB). Report of the 10th Meeting. Geneva, World Health Organization, 2010.

21 World Health Organization. Checklist of Prerequisites to Country Implementation of Xpert MTB/RIF and Key Action Points at Country Level. Geneva, World Health Organization, 2011. 
22 World Health Organization and The Stop TB Partnership. The Global Plan to Stop TB, 2011-2015. Transforming the Fight Towards Elimination of Tuberculosis. Geneva, World Health Organization, 2010.

23 Chang $\mathrm{K}, \mathrm{Lu} \mathrm{W}$, Wang J, et al. Rapid and effective diagnosis of tuberculosis and rifampicin resistance with Xpert MTB/RIF assay: a meta-analysis. J Infect 2012; 64: 580-588.

24 Van Rie A, Page-Shipp L, Scott L, et al. Xpert® MTB/RIF for point-of-care diagnosis of TB in high-HIV burden, resource-limited countries: hype or hope? Expert Rev Mol Diagn 2010; 10: 937-946.

Small P, Pai M. Tuberculosis diagnosis - time for a game change. N Engl J Med 2010; 363: 1070-1071.

Evans CA. GeneXpert - a game-changer for tuberculosis control? PLoS Med 2011; 8: e1001064.

Zeka AN, Tasbakan S, Cavusoglu C. Evaluation of the GeneXpert MTB/RIF assay for the rapid diagnosis of tuberculosis and detection of RIF-resistance in pulmonary and extrapulmonary specimens. J Clin Microbiol 2011; 49: 4138-4141.

28 Hanif SN, Hana ES, Suhail A, et al. GeneXpert® MTB/RIF for rapid detection of Mycobacterium tuberculosis in pulmonary and extra-pulmonary samples. Int J Tuberc Lung Dis 2011; 15: 1274-1275.

29 Ioannidis P, Papaventsis D, Karabela S, et al. Cepheid GeneXpert MTB/RIF assay for Mycobacterium tuberculosis detection and rifampin resistance identification in patients with substantial clinical indications of tuberculosis and smear-negative microscopy results. J Clin Microbiol 2011; 49: 3068-3070.

30 Teo J, Jureen R, Chiang D, et al. Comparison of two nucleic acid amplification assays, the Xpert MTB/RIF and the amplified Mycobacterium Tuberculosis Direct (MTD) assay, for the detection of Mycobacterium tuberculosis in respiratory and non-respiratory specimens. J Clin Microbiol 2011; 49: 3659-3662.

31 Miller MB, Popowitch EB, Michael G, et al. Performance of Xpert MTB/RIF assay and IS6110 real-time PCR for Mycobacterium tuberculosis detection in clinical samples. J Clin Microbiol 2011; 49: 3458-3462.

32 Blakemore R, Nabeta P, Davidow AL, et al. A multi-site assessment of the quantitative capabilities of the Xpert MTB/RIF assay. Am J Respir Crit Care Med 2011; 184: 1076-1084.

33 Scott LE, McCarthy K, Gous N, et al. Comparison of Xpert MTB/RIF with other nucleic acid technologies for diagnosing pulmonary tuberculosis in a high HIV prevalence setting: a prospective study. PLoS Med 2011; 8: e1001061.

34 Theron G, Peter J, van Zyl-Smit R, et al. Evaluation of the Xpert MTB/RIF assay for the diagnosis of pulmonary tuberculosis in a high HIV prevalence setting. Am J Respir Crit Care Med 2011; 184: 132-140.

35 Lawn SD, Brooks SV, Kranzer K, et al. Screening for HIV-associated tuberculosis and rifampicin resistance before antiretroviral therapy using the Xpert MTB/RIF assay: a prospective study. PLoS Med 2011; 8: e1001067.

36 Bowles EC, Freyée B, van Ingen J, et al. Xpert MTB/RIF®, a novel automated polymerase chain reaction-based tool for the diagnosis of tuberculosis. Int J Tuberc Lung Dis 2011; 15: 988-989.

37 Rachow A, Zumla A, Heinrich N, et al. Rapid and accurate detection of Mycobacterium tuberculosis in sputum samples by Cepheid Xpert MTB/RIF assay-a clinical validation study. PLoS One 2011; 6: e20458

38 Armand S, Vanhuls P, Delcroix G, et al. Comparison of the Xpert MTB/RIF test with an IS6110-TaqMan real-time PCR assay for direct detection of Mycobacterium tuberculosis in respiratory and non respiratory specimens. J Clin Microbiol 2011; 49: 1772-1776.

39 Malbruny B, Le Marrec G, Courageux K, et al. Rapid and efficient detection of Mycobacterium tuberculosis in respiratory and non-respiratory samples. Int J Tuberc Lung Dis 2011; 15: 553-555.

40 Marlowe EM, Novak-Weekley SM, Cumpio J, et al. Evaluation of the Cepheid Xpert MTB/RIF assay for direct detection of Mycobacterium tuberculosis complex in respiratory specimens. J Clin Microbiol 2011; 49: 1621-1623.

41 Moure R, Muñoz L, Torres M, et al. Rapid detection of Mycobacterium tuberculosis complex and rifampin resistance in smear-negative clinical samples by use of an integrated real-time PCR method. J Clin Microbiol 2011; 49: 1137-1139.

42 Trébucq A, Enarson EA, Chiang CY, et al. Xpertæ MTB/RIF for national tuberculosis programmes in low-income countries: when, where and how? Int J Tuberc Lung Dis 2011; 15: 1567-1572.

43 Singh JA, Bhan A. The ethics of national tuberculosis programmes in low-income countries not rolling out Xpert $\mathbb{R}$ MTB/RIF. Int J Tuberc Lung Dis 2011; 15: 1563.

44 Lawn SD, Nicol MP. Xpert $\mathbb{B}$ MTB/RIF assay: development, evaluation and implementation of a new rapid molecular diagnostic for tuberculosis and rifampicin resistance. Future Microbiol 2011; 6: 1067-1082.

45 Dowdy DW, Cattamanchi A, Steingart KR, et al. Is scale-up worth it? Challenges in economic analysis of diagnostic tests for tuberculosis. PLoS Med 2011; 8: e1001063.

46 Meyer-Rath G, Bistline K, Long L, et al. The Incremental Cost of Introducing Xpert ${ }^{8}$ MTB/RIF into the South African National Tuberculosis Programme: Results of the National TB Cost Model 2011/12-2016/17. Johannesburg, Health Economics and Epidemiology Research Office, 2011.

47 Salvo F, Sadutshang TD, Migliori GB, et al. Xpert MTB/RIF test for tuberculosis. Lancet 2011; 378: 481-482.

48 Ferrara G, O’Grady J, Zumla A, et al. Xpert MTB/RIF test for tuberculosis. Lancet 2011; 378: 482.

49 Theron G, Peter J, Dheda K. Xpert MTB/RIF test for tuberculosis. Lancet 2011; 378: 481.

50 Nicol MP, Workman L, Isaacs W, et al. Accuracy of the Xpert MTB/RIF test for the diagnosis of pulmonary tuberculosis in children admitted to hospital in Cape Town, South Africa: a descriptive study. Lancet Infect Dis 2011; 11: 819-824.

51 Van Zyl-Smit RN, Binder A, Meldau R, et al. Comparison of quantitative techniques including XpertMTB/RIF to evaluate mycobacterial burden. PLoS One 2011; 6: e28815.

52 Friedrich SO, von Groote-Bidlingmaier F, Diacon AH. Xpert MTB/RIF assay for the diagnosis of pleural tuberculosis. J Clin Microbiol 2011; 49: 4341-4342.

53 Causse M, Ruiz P, Gutiérrez-Aroca JB, et al. Comparison of two molecular methods for rapid diagnosis of extrapulmonary tuberculosis. J Clin Microbiol 2011; 49: 3065-3067.

54 Ligthelm LJ, Nicol MP, Hoek KGP, et al. Xpert ${ }^{\circledR}$ MTB/RIF for the rapid diagnosis of tuberculous lymphadenitis from fine needle aspiration biopsy specimens. J Clin Microbiol 2011; 49: 3967-3970.

55 Vadwai V, Boehme C, Nabeta P, et al. Xpert MTB/RIF: a new pillar in diagnosis of extrapulmonary tuberculosis? J Clin Microbiol 2011; 49: 2540-2545.

56 Hillemann D, Rüsch-Gerdes S, Boehme C, et al. Rapid molecular detection of extrapulmonary tuberculosis by the automated GeneXpert MTB/RIF system. J Clin Microbiol 2011; 49: 1202-1205.

57 Theron G, Pooran A, Peter J, et al. Do adjunct tuberculosis tests, when combined with Xpert MTB/RIF, improve accuracy and the cost of diagnosis in a resource-poor setting? Eur Respir J 2012; 40: 161-168. 


\section{Infect Ther 2012; 10: 631-635.}

60 Taylor N, Gaurb RL, Ellen J, et al. Can a simple flotation method lower the limit of detection of M. tuberculosis in extrapulmonary samples by the GeneXpert MTB/RIF assay? J Clin Microbiol 2012; 50: 2272-2276.

61 Lawn SD, Kerkhoff AD, Vogt M, et al. High diagnostic yield of tuberculosis from screening urine samples from HIV-infected patients with advanced immunodeficiency using the Xpert MTB/RIF assay. J Acquir Immune Defic Syndr 2012; 60: 289-294.

62 Moure R, Martína R, Alcaide F. Effectiveness of an integrated real-time PCR method for detection of the Mycobacterium tuberculosis complex in smear-negative extrapulmonary samples in an area of low tuberculosis prevalence. J Clin Microbiol 2012; 50: 513-515.

63 O'Grady J, Bates M, Chilukutu L, et al. Evaluation of the Xpert MTB/RIF assay at a tertiary care referral hospital in a setting where tuberculosis and HIV infection are highly endemic. Clin Infect Dis 2012; 55: 1171-1178.

64 Peter JG, Theron G, Muchinga TE, et al. The diagnostic accuracy of urine-based Xpert MTB/RIF in HIV-infected hospitalized patients who are smear-negative or sputum scarce. PLoS One 2012; 7: e39966.

65 Williamson DA, Basu I, Bower J, et al. An evaluation of the Xpert MTB/RIF assay and detection of false-positive rifampicin resistance in Mycobacterium tuberculosis. Diagn Microbiol Infect Dis 2012; 74: 207-209.

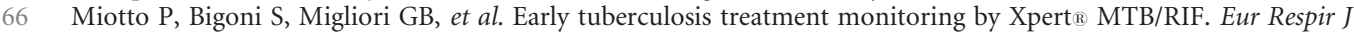
2012; 39: 1269-1271.

67 Lawn SD, Kerkhoff AD, Vogt M, et al. Characteristics and early outcomes of patients with Xpert MTB/RIF-negative pulmonary tuberculosis diagnosed during screening before antiretroviral therapy. Clin Infect Dis 2012; 54: 1071-1079.

68 Theron G, Peter J, Dheda K. Characteristics of Xpert MTB/RIF-negative patients with pulmonary tuberculosis. Clin Infect Dis 2012; 55: 472-472.

69 Theron G, Pinto L, Peter J, et al. The use of an automated quantitative polymerase chain reaction (Xpert MTB/RIF) to predict the sputum smear status of tuberculosis patients. Clin Infect Dis 2012; 54: 384-388.

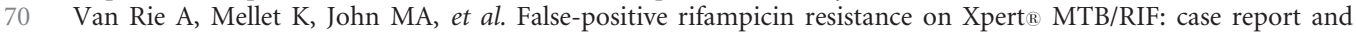
clinical implications. Int J Tuberc Lung Dis 2012; 16: 206-208.

71 Rachow A, Clowes P, Saathoff E, et al. Increased and expedited case detection by Xpert MTB/RIF assay in childhood tuberculosis: a prospective cohort study. Clin Infect Dis 2012; 54: 1388-1396.

72 Tortoli E, Russo C, Piersimoni C, et al. Clinical validation of Xpert MTB/RIF for the diagnosis of extrapulmonary tuberculosis. Eur Respir J 2012; 40: 442-447.

73 Zar HJ, Workman L, Isaacs W, et al. Rapid molecular diagnosis of pulmonary tuberculosis in children using nasopharyngeal specimens. Clin Infect Dis 2012; 55: 1088-1095.

74 Turnbull E, Kancheya NG, Harris JB, et al. A model of tuberculosis screening for pregnant women in resourcelimited settings using Xpert MTB/RIF. J Pregnancy 2012; 2012: 565049.

75 Meyer-Rath G, Schnippel K, Long L, et al. The impact and cost of scaling up GeneXpert MTB/RIF in South Africa. PLoS One 2012; 7: e36966.

76 Schnippel K, Meyer-Rath G, Long L et al. Scaling up Xpert MTB/RIF technology: the costs of laboratory- vs. clinicbased roll-out in South Africa. Trop Med Int Health 2012; 17: 1142-1151.

77 Andrews JR, Lawn SD, Rusu C, et al. The cost-effectiveness of routine tuberculosis screening with Xpert MTB/RIF prior to initiation of antiretroviral therapy in South Africa: a model-based analysis. J Acquired Immune Defic Syndr 2012; 26: 987-995.

78 Abimbola TO, Marston BJ, Date AA. Cost-effectiveness of tuberculosis diagnostic strategies to reduce early mortality among persons with advanced HIV infection initiating antiretroviral therapy. J Acquired Immune Defic Syndr 2012; 60: e1-e7.

79 Peters D, Theron G, Peter J, et al. Should Xpert® MTB/RIF be rolled out in low-income countries? Int J Tuberc Lung Dis 2012; 16: 702-703.

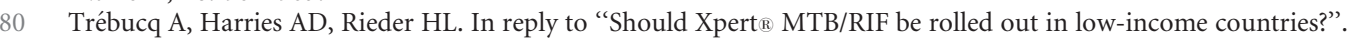
Int J Tuberc Lung Dis 2012; 16: 703-704.

81 Lawn SD, Kerkhoff AD, Wood R, et al. Location of Xpert® MTB/RIF in centralised laboratories in South Africa undermines potential impact. Int J Tuberc Lung Dis 2012; 16: 701.

82 Trébucq A, Harries AD. In reply to "Location of Xpert $\mathbb{B}$ MTB/RIF in centralised laboratories in South Africa undermines potential impact”. Int J Tuberc Lung Dis 2012; 16: 702.

83 Bodmer TA. Ströhle A.: Diagnosing pulmonary tuberculosis with the Xpert MTB/RIF test. J Vis Exp $2012 ; 62$ : e3547.

84 World Health Organization and Global Laboratory Initiative (GLI). 4th Annual GLI Meeting/Consultation of the WHO/GLI SRL Network/Early Implementers Meeting on Xpert MTB/RIF roll-out, Annecy, Stop TB Partnership, 2012.

85 Pantoja A, Fitzpatrick C, Vassall A, et al. Xpert MTB/RIF for the rapid diagnosis of TB and drug-resistant TB: a cost and affordability analysis. Eur Respir J 2012 [In press DOI: 10.1183/09031936.00147912].

86 Dorman SE, Chihota VN, Lewis JJ, et al. Performance characteristics of the Cepheid Xpert MTB/RIF test in a tuberculosis prevalence survey. PLoS One 2012; 7: e43307.

87 Kirwan DE, Cardena MK, Gilman RH. Rapid implementation of new TB diagnostic tests: is it too soon for a global roll-out of Xpert MTB/RIF? Am J Trop Med Hyg 2012; 87: 197-201.

88 Talbot EA, Pape J, Sundaram L, et al. Transforming TB diagnosis: can patients and control programs afford to wait? Am J Trop Med Hyg 2012; 87: 202-204.

89 Cobelens F, van den Hof S, Pai M, et al. Which new diagnostics for tuberculosis, and when? J Infect Dis 2012; 205: Suppl. 2, S191-S198.

90 Pai NP, Pai M. Point-of-care diagnostics for HIV and tuberculosis: Landscape, pipeline, and unmet needs. Discov Med 2012; 13: 35-45.

91 USAID. Press release: Public-Private Partnership Announces Immediate 40 Percent Cost Reduction for Rapid TB Test. August 6, 2012. www.usaid.gov/news-information/press-releases/public-private-partnership-announcesimmediate-40-percent-cost

92 World Health Organization. Policy Framework for Implementing TB Diagnostics. Geneva, World Health Organization, 2011. 
93 World Health Organization. Guidelines for the Programmatic Management of Drug-resistant Tuberculosis. Geneva, World Health Organization, 2008.

94 World Health Organization. Guidelines for the Programmatic Management of Drug-resistant Tuberculosis: 2011 Update. Geneva, World Health Organization, 2011.

95 World Health Organization. WHO Policy on Collaborative TB/HIV Activities: Guidelines for National Programmes and Other Stakeholders. Geneva, World Health Organization, 2012.

96 Getahun H, Kittikraisak W, Heilig CM, et al. Development of a standardized screening rule for tuberculosis in people living with HIV in resource-constrained settings: individual participant data meta-analysis of observational studies. PLoS Med 2010; 8: e1000391.

97 Van Deun A, Barrera L, Bastian S, et al. Mycobacterium tuberculosis strains with highly discordant rifampin susceptibility test results. J Clin Microbiol 2009; 47: 3501-3506.

98 World Health Organization. Treatment of Tuberculosis: Guidelines for National Programmes. 4th Edn. Geneva, World Health Organization, 2009.

99 Falzon D, Jaramillo E, Schünemann HJ, et al. WHO guidelines for the programmatic management of drug-resistant tuberculosis: 2011 update. Eur Respir J 2011; 38: 516-528.

100 Zignol M, van Gemert W, Falzon D, et al. Modernizing surveillance of anti-tuberculosis drug resistance: from special surveys to routine testing. Clin Inf Dis 2011; 52: 901-906.

101 Chonde TM, Doulla B, van Leth F, et al. Implementation of the tuberculosis drug resistance survey in Tanzania. BMC Public Health 2008; 8: 427.

102 Smith SE, Kurbatova EV, Cavanaugh JS, et al. Global isoniazid resistance patterns in rifampin-resistant and rifampin-susceptible tuberculosis. Int J Tuberc Lung Dis 2012; 16: 203-205.

103 World Health Organization. Guidelines on Ethics of Tuberculosis Prevention, Care and Control. Geneva, World Health Organization, 2010

104 Havlir D, Beyrer C. The beginning of the end of AIDS? N Engl Med J 2012; 367: 685-687.

105 Jiménez-Levi E. 2011 Report on Tuberculosis Research Funding Trends, 2005-2010. 2nd Edn. New York, Treatment Action Group/Stop TB Partnership, 2012.

106 Kim SY, Kim H, Ra EK, et al. The Xpert ${ }^{\circ}$ MTB/RIF assay evaluation in South Korea, a country with an intermediate tuberculosis burden. Int J Tuberc Lung Dis 2012; 16: 1471-1476.

107 Barnard M, Gey van Pittius NC, van Helden PD, et al. Diagnostic performance of Genotype MTBDRplus version 2 line probe assay is equivalent to the Xpert巴MTB/RIF assay. J Clin Microbiol 2012; 50: 3712-3716.

108 Carriquiry G, Otero L, González-Lagos E, et al. Diagnostic accuracy study of Xpert $\mathbb{R}$ MTB/RIF in HIV-positive patients with high clinical suspicion of pulmonary tuberculosis in Lima, Peru. PLoS One 2012; 7: e44626.

109 Alvarez-Uria G, Azcona JM, Midde M, et al. Rapid Diagnosis of pulmonary and extrapulmonary tuberculosis in HIV infected patients. comparison of LED fluorescent microscopy and the GeneXpert MTB/RIF assay in a district hospital in India. Tuberc Res Treat 2012; 2012: 932862.

110 Ntinginya EN, Squire SB, Millington KA, et al. Performance of the Xpert $\mathbb{B}$ MTB/RIF assay in an active case-finding strategy: a pilot study from Tanzania. Int J Tuberc Lung Dis 2012; 16: 1468-1470.

111 Balcells ME, García P, Chanqueo L, et al. Rapid molecular detection of pulmonary tuberculosis in HIV-infected patients in Santiago, Chile. Int J Tuberc Lung Dis 2012; 16: 1349-1353.

112 Whittaker E, Zar HJ. Promising directions in the diagnosis of childhood tuberculosis. Expert Rev Respir Med 2012; 6: 385-395. 